\title{
TWO NEW BUCEPHALIDAE (TREMATODA) FROM FISHES OF THE BRAZILIAN AMAZON
}

\section{Vernon E. THATCHER'}

\begin{abstract}
Two new species of Bucephalidae (Trematoda) are described from fishes of the Brazilian Amazon. Glandulorhynchus turgidus gen, et sp. n. from Hydrolycus sp. differs from all others in the family by having conspicuous glands near the anterior extremity and an expanded uterus used for storing eggs. Prosorhynchus piranhus sp. n. from Serrasalmus rhombeus is characterized as having a small rhynchus and pharynx, and a large cirrus sac.
\end{abstract}

Key words: trematodes, fish parasites, bucephalids, Brazil, Amazonia.

Duas Novas Espécies de Bucephalidae (Trematoda) de Peixes da Amazônia Brasileira

RESUMO - Duas novas espécies de Bucephalidae (Trematoda) são descritas de peixes da Amazônia brasileira. Glandulorhynchus turgidus gen. et sp. n. parasita de Hydrolycus sp. distingue-se das demais espécies da família por apresentar glândulas conspícuas próximo à extremidade anterior e um útero expandido para amazenar ovos. Prosorhynchus piranhus sp. $\mathrm{n}$. parasita de Serrasalmus rhombeus é caracterizado por ter um "rhynchus" pequeno e uma bolsa de cirro grande.

Palavras chaves: trematódeos, parasitas de peixes, bucefalideos, Brasil, Amazônia.

\section{INTRODUÇÃO}

The Bucephalidae constitute a small family of trematodes found mainly in marine fishes. Thatcher (1993) listed nine genera and 28 species from the Neotropical Region but only five of these have been reported from freshwater. In Brazil, the bucephalids are known principally through the publications of Kohn (1961a; 1961b; 1962a; 1962b; 1963; 1966; 1967a; 1967b; $1968 ; 1970 \mathrm{a} ; 1970 \mathrm{~b} ; 1970 \mathrm{c})$. In the present work, two new species, one of which is a new genus, are described.

\section{MATERIALS AND METHODS}

Fish hosts were netted, identified and eviscerated in the field. Their intestinal tracts were removed, fixed in $10 \%$ formalin solution and later transferred to $70 \%$ ethanol. At the laboratory in Manaus, the preserved digestive tracts were opened and the contents were examined by washing and hand sedimentation. Whole mount preparations were made by means of the phenol/balsam method explained in Thatcher (1991). Drawings were made with the aid of a Zeiss drawing tube. Measurements were made with a measuring ocular and mean sizes are followed by the extremes in parentheses. All sizes are in micrometers $(\mu \mathrm{m})$ unless indicated as millimeters ( $\mathrm{mm})$.

RESULTS

\section{Systematic Section}

Family Bucephalidae Poche, 1907

\section{Glandulorhynchus gen. $\mathbf{n}$.}

Generic diagnosis: Body stout, flattened, unspined; rhynchus suckerlike, provided with numerous glands. Pharynx small, near equator or poste-

Instituto Nacional de Pesquisas da Amazônia, Manaus, AM, Brazil. Research, fellow of the Brazilian National Research Council (CNPq). 
rior thereto; gut saccular, mostly posterior to pharynx. Testes two, spherical, diagonal, posterior to pharynx; cirrus sac small, at posterior extremity; genital pore subterminal. Ovary spherical, anterior to pharynx; vitellaria in grape-like clusters, extending anterolaterally in anterior half of body; uterus tubular forming massive uterine reservoir on side of body; eggs small, numerous. Intestinal parasites of freshwater fishes.

Type species: Glandulorhynchus turgidus $\mathrm{sp} . \mathrm{n}$.

\section{Glandulorhynchus turgidus sp. n.} (Figs. 1, 2)

Host: Hudrolycus Müller \&
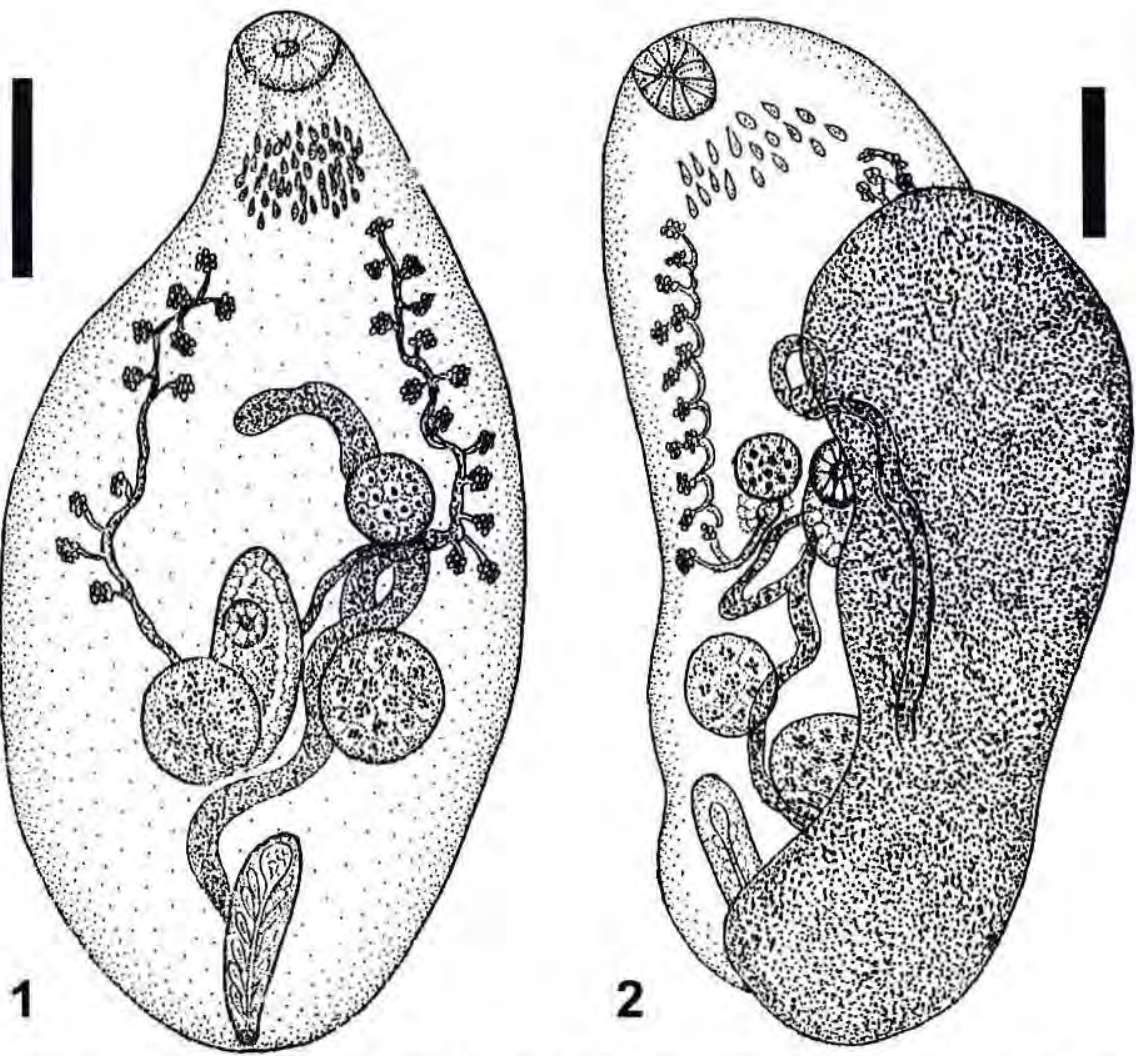

Figures 1, 2. Glandulorhynchus turgidus gen. et sp. nov. (ventral view), scale bars $=1 \mathrm{~mm}$. 
Species description (10 specimens measured): With the characters of the genus. Body 6.5 (5.0-7.3) mm long and $3.0(2.5-3.5) \mathrm{mm}$ wide. Rhynchus 629 (562-811) long by 630 (541-811) wide; glands associated with rhynchus 131-174 long and 8789 wide. Pharynx 276 (208-312) long by 288 (250-354) wide; gut 1.4 (0.61.9) $\mathrm{mm}$ long and 535 (461-624) in diameter. Anterior testis 0.84 (0.6-1.0) $\mathrm{mm}$ long and $0.7(0.5-0.8) \mathrm{mm}$ in width; posterior testis $0.8(0.6-1.0)$ $\mathrm{mm}$ long and 0.7 (0.6-0.8) $\mathrm{mm}$ wide; cirrus sac $1.3(1.2-1.5) \mathrm{mm}$ long and $335(270-416)$ in diameter. Ovary 418 (354-478) long and 404 (354-416) wide; vitelline glands measure 40-60, clusters of glands 166-205; uterine reservoir measures $4.5-6.0 \mathrm{~mm}$ long by $2.0-2.5 \mathrm{~mm}$ wide; eggs measure 19 x 10 (18-20 x 9-11).

Remarks: Glandulorhynchus turgidus gen. et $\mathrm{sp}$. $\mathrm{n}$. is not closely similar to any other known bucephalid trematode. It has three unique features, namely: 1) glands in the anterior region associated with the rhynchus; 2) grape like vitellaria; and 3) a large uterine reservoir.

\section{Prosorhynchus Odhner, 1905}

\section{Prosorhynchus piranhus sp. n. (Fig. 3)}

Host: Serrasalmus rhombeus (Linneaus, 1766).

Site: Intestinal tract.

Locality: Capucapu River, Amazonas State, Brazil.

Holotype (INPA-369) and 5 paratypes (INPA-370a-e): Invertebrate Collection of the Instituto Nacional de Pesquisas da Amazônia,
Manaus, AM, Brazil.

Colector: M. Jégu, 30/9/88.

Etymology: The specific name comes from the common name of the host fish (piranha).

Specific diagnosis ( 6 specimens measured): Body subspherical measures $963(783-1,088)$ long and 635 (422-748) wide. Rhynchus small, measures 93 (66-119) long and 112 (88-132) wide. Pharynx small, 76 (66-83) long by 77 (66-83) wide; gut saccular, projects anteriorly from pharynx, measures $149(88-198)$ long and $95(73-110)$ wide. Testes, subspherical, tandem, lateral andposterolateral to pharynx; anterior testis measures 113 (101-132) long and $88(77-110)$ wide; posterior testis 121 (110-132) long and 103 (88-117) wide; cirrus sac measures 362 (176-487) long and 97 (66-113) in diameter. Ovary subspherical, lateral to pharynx, pretesticular, measures 90 (57-125) long and 77 (50-99) wide; vitellaria consist of few spherical follicles centrally situated, pre-equatorial, measure 26-44 in diameter; eggs measure $48 \times 25$ ( $44-55 \times 22-31)$.

Remarks: The species attributed to the genus Prosorhynchus differ considerably among themselves. Some species have a sucker-like rhynchus and in others that organ is plug-like. The type species of the genus, $P$. squamatus Odhner, 1905, described from a marine fish, has a suckerlike rhynchus and somewhat resembles the new Amazonian species. P. piranhus sp. n. differs from the type species in having a more compact, subspherical body, vitellaria far from the anterior extremity and a larger cirrus sac. 


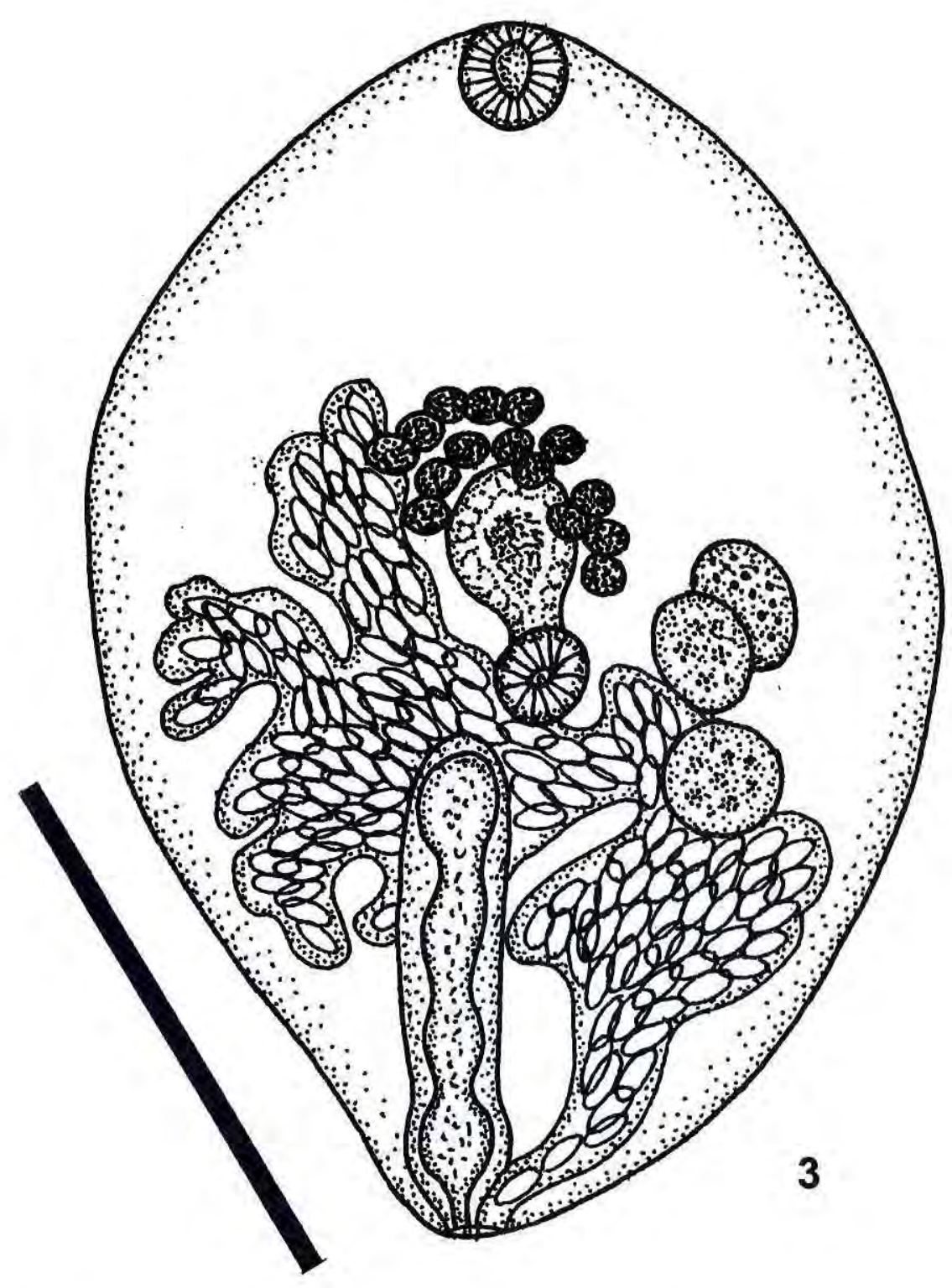

Figure 3. Prosorhynchus piranhus sp. nov. (ventral view). scale $=500 \mu \mathrm{m}$. 


\section{Literature Cited}

Kohn, A. 1961a. Um novo Rhipidocotyle parasito de Scomberomerus maculatus (Hitch.) (Trematoda, Bucephaliformes). Atas Soc. Biol. Rio de Janeiro, 5: 41-44.

Kohn, A. 1961b. Um novo Prosorhynchus parasito de Garupa sp. (Trematoda, Bucephaliformes. Atas Soc. Biol. Rio de Janeiro, 5: 46-49.

Kohn, A. 1962a. Nota prévia sobre uma nova espécie do gênero Bucephalopsis Diesing, 1855 (Trematoda, Bucephaliformes). Atas Soc. Biol. Rio de Janeiro, 6: 14-18.

Kohn, A. 1962b. Sobre um novo género de trematódeo bucefaliforme parasito de peixe de água doce. Rev. Brasil. Biol., 22: 351-355.

Kohn, A. 1963. Paurorhynchus schubarti sp. n., metacercária parasita de dourado (Trematoda, Bucephaliformes). Rev. Brasil. Biol.. 23: 259-261.

Kohn, A. 1966. Bucephalus solitarius sp. n., parasito de peixe do litoral brasileiro (Trematoda, Bucephaliformes). Atas Soc. Biol. Rio de Janeiro, 10: 87-90.

Kohn, A. 1967a. Ocorrência de Prosorhynchus ozakii Manter, 1934 (Trematoda, Bucephaliformes) em novo hospedador da Bahia de Guanabara. Atas Soc. Biol. Rio de Janeiro. 11: 69-70.
Kohn, A. 1967b. Sobre um novo gênero de Prosorhynchinae Nicoll, 1914 e novos dados sobre Prosorhynchus bulbosus Kohn, 1961, e Rhipidocotyle quadriculatum Kohn, 1961 (Trematoda, Bucephaliformes). Mem. Inst. Oswaldo Cruz, 65: 107-114.

Kohn,A. 1968. Ocorrência de Bucephalus varicus Manter, 1940 (Trematoda, Bucephaliformes) na Bahia de Guanabara. Atas Soc. Biol. Rio de Janeiro, 11: 165-166.

Kohn, A. 1970a. Chabaudtrema rarus gen, n., sp. n., trematódeo bucefaliforme parasito de peixe. Atas Soc. Biol. Rio de Janeiro, 13: 147-148.

Kohn, A. 1970b. Pararhipidocotyle jeffersoni gen. n., sp. n., trematódeo bucefaliforme parasito de dourado. Atas Soc. Biol. Rio de Janeiro, 13: 181-183.

Kohn, A. 1970c. Bellumcorpus schubarti (Kohn, 1963) comb. nov. para Paurorhynchus schubarti Kohn, 1963 (Trematoda, Prosorhynchidae). Atas Soc. Biol. Rio de Janeiro, 13: 185-186.

Thatcher, V.E. 1991. Amazon fish parasites. Amazoniana. 11: 263-571.

Thatcher, V.E. 1993. Trematódeos Neotropicais. INPA. Manaus. $553 \mathrm{p}$. 